Q.-H. Wei

C. Bechinger

P. Leiderer

\title{
Experimental observation of single-file diffusion of Brownian particles
}

\begin{abstract}
The self-diffusion of particles restricted to one-dimensional channels is termed single-file diffusion if individual particles are unable to pass each other. We employ the interference pattern of two coherent laser beams to confine electrically charged polystyrene colloids in parallel "optical channels" formed by light forces and study the particle diffusion within these channels by optical microscopy. For small channel spacings the particles can electrically couple between adjacent
\end{abstract}

channels and their mean square displacement along these channels at long observation times $t$ is proportional to $t$. For large channel spacings, however, where the coupling of particles is largely reduced, we find the mean square displacement to be proportional to $t^{0.5}$, which signifies the occurrence of single-file diffusion.

Key words Single file diffusion Brownian particles - Colloids Light forces
Faculty of Physics

University of Konstanz

Postfach M676, D-78457 Konstanz

Germany

$$
\left\langle x^{2}(t)\right\rangle=2 D t
$$

For short times $\left(t \gg a^{2} / 2 D_{0}\right)$, where the tagged particle moves in the cage formed by neighboring particles, $D$ is called the short-time self-diffusion coefficient $D_{\mathrm{S}}$, which, for very dilute particle concentration, equals $D_{0}$, the diffusion coefficient of a free particle of diameter $a$. In contrast, for long times $\left(t \gg a^{2} / 2 D_{0}\right)$, a particle feels the presence of other particles and mutual interactions reduce the diffusion coefficient; $D$ is thus called the long-time self-diffusion coefficient $D_{\mathrm{L}}$. The mean square displacement of single-file diffusion, however, has been shown theoretically to increase with the square root of time $[2-4]$ :

$\left\langle x^{2}(t)\right\rangle=2 F t^{0.5}$

where $F$ is the single-file mobility.

Although numerous theoretical investigations on this topic were performed in the past, only recently has single-file diffusion became experimentally accessible. A crystalline zeolite such as $\mathrm{AlPO}_{4}-5$, which contains long unconnected parallel channels with a diameter of $7.3 \AA$, 
provides a good single-file system for adsorbates like methane whose diameter is $3.8 \AA$. In pulsed force gradient nuclear magnetic resonance (PFG NMR) experiments where the transport of methane and ethane in these molecular sieves has been studied, evidence for single-file diffusion has been found [8-11].

These results are not in agreement with very recent quasi-elastic neutron scattering studies (QENS), however, which show that both methane and ethane diffuse normally in $\mathrm{AlPO}_{4}-5$ molecular sieves [12]. Several possible reasons have been proposed to explain this controversy. Firstly, owing to defects in zeolites the nano-channels in these materials are not infinitely long. Additionally, some correlation between molecules in neighboring channels may exist, as suggested to explain the experimentally observed phase transition in this onedimensional system [13]. However, no detailed comparison between theory and experimental results can be made owing to the lack of the real space information of these adsorbate molecules. Therefore, it is of great interest to perform self-consistent experimental studies which can provide real space information of particles.

A monodisperse colloidal dispersion has been proven to be a unique model system, not only because of its similarity to atomic systems but also due to its characteristic length and time scales which make colloids accessible to optical microscopy [14]. In this paper, we studied the diffusion behavior of charged monodisperse polystyrene colloids which are confined between two glass plates. To restrict the diffusion of the particles to one dimension, we projected the interference pattern of two coherent laser beams into the sample cell. Owing to optical gradient forces these dielectric colloidal beads are then trapped in the regions of highest intensity, i.e. along a pattern of parallel lines. In this paper we will demonstrate that the line spacing plays a crucial role for the occurrence of single-file diffusion. For low line spacings the particle mean square displacement along these pores is found to increase proportional to $t$ because the particles in neighboring lines do not diffuse independently but are coupled due to electrostatic interactions. Only when the spacing of adjacent lines is large enough and electrostatic interactions across lines are largely reduced does the mean square displacement increase as $t^{0.5}$, which is in agreement with single-file diffusion.

\section{Experimental}

The experimental system has been described before [15]. We used an aqueous suspension of highly charged polystyrene colloidal particles with $3 \mu \mathrm{m}$ diameter (IDC). The sample cell was composed of two glass plates which were separated by about $20 \mu \mathrm{m}$. This narrow spacing is important to suppress thermal convection, which is observed for spacings above a few hundred microns owing to heating effects [16]. The tray ions in the cell are removed by pumping the suspension through a circuit containing an ion exchange vessel and an ionic conductivity probe. Additionally, a certain amount of ion exchange resin particles were added to the edge of the cell to stabilize our system at low salt concentration during the experiments.

We used a setup similar to the Mach-Zender interferometer to produce two parallel coherent laser beams with separation $D$ from an argon ion laser (TM00 mode, $\lambda=514 \mathrm{~nm}$ ). The beams were introduced to cross by a convergent lens and produced interference fringes in the plane of the horizontally aligned sample. The fringe spacing was adjusted by the spacing $D$ which could be varied by the position of a motor-driven mirror. An optical microscope system attached to a CCD camera was used to observe the particles. White light was used to illuminate the particles while the scattered intense laser light was blocked with an optical high-pass filter. The images were stored on videotape for further analysis.

As illustrated by Ashkin et al. [17], the forces exerted by the laser field on dielectric microparticles can be divided into two main components. One, proportional to the light intensity, is the scattering force that drives the particle in the direction of the light, which in our system pushes the colloidal spheres into the bottom glass plate and reduces particle fluctuation in the vertical direction, making the system two dimensional. The other component, proportional to intensity gradient, is the gradient force due to diffraction which pulls the polystyrene particles into the regions of high light intensity. The latter force from the parallel interference fringes in our system results in a one-dimensional periodic external potential for the colloidal spheres. With the particle finite size taken into account, the modulation potential $V(x)$ can be expressed as [16]:

$V(x)=-V_{0} \cos (2 \pi x / d)$

where $V_{0}=\left[3 n_{w} P \sigma_{0}^{3}\left(n^{2}-1\right) / c r_{0}^{2}\left(n^{2}+2\right)\right]\left[j_{1}\left(\pi \sigma_{0} / d\right) d / 2 \pi \sigma_{0}\right]$, with $P$ being the laser power, $c$ the velocity of light in a vacuum, $n$ the ratio of the refraction indices of polystyrene $n_{\mathrm{p}}$ and water $n_{\mathrm{w}}, \sigma_{0}$ the colloidal particle diameter, $j_{1}$ the first-order spherical Bessel function, $r_{0}$ the waist radius of the Gaussian laser beam, and $d$ the period of the potential corresponding to the fringe spacing.

\section{Results}

Figure la shows a typical real space configuration of particles in our cell under the influence of an external periodic light potential. Owing to the external potential, the particles are not homogeneously distributed but localized along parallel lines. This behavior indicates that the external light potential provides a lateral confinement of the particles and thus mimics, for example, the pores in a zeolitic system. It is important to mention that the line spacing in Fig. 1a is about $11 \mu \mathrm{m}$, which is within the interaction range of particles. Accordingly, particles in neighboring lines diffuse not independently from each other but are highly correlated owing to the repulsive Coulomb interaction. This is visible in more detail in the corresponding particle distribution function $g(x, y)$ averaged over 200 pictures, which clearly shows a hexagonal symmetry (Fig. 1b).

To analyze the diffusion behavior of the particles, we calculated the mean square displacement $\left\langle\delta y^{2}\right\rangle$ of particles along the lines. Figure 2 shows the results for systems with high (closed symbols) and low (open symbols) particle concentrations. It can be seen that 

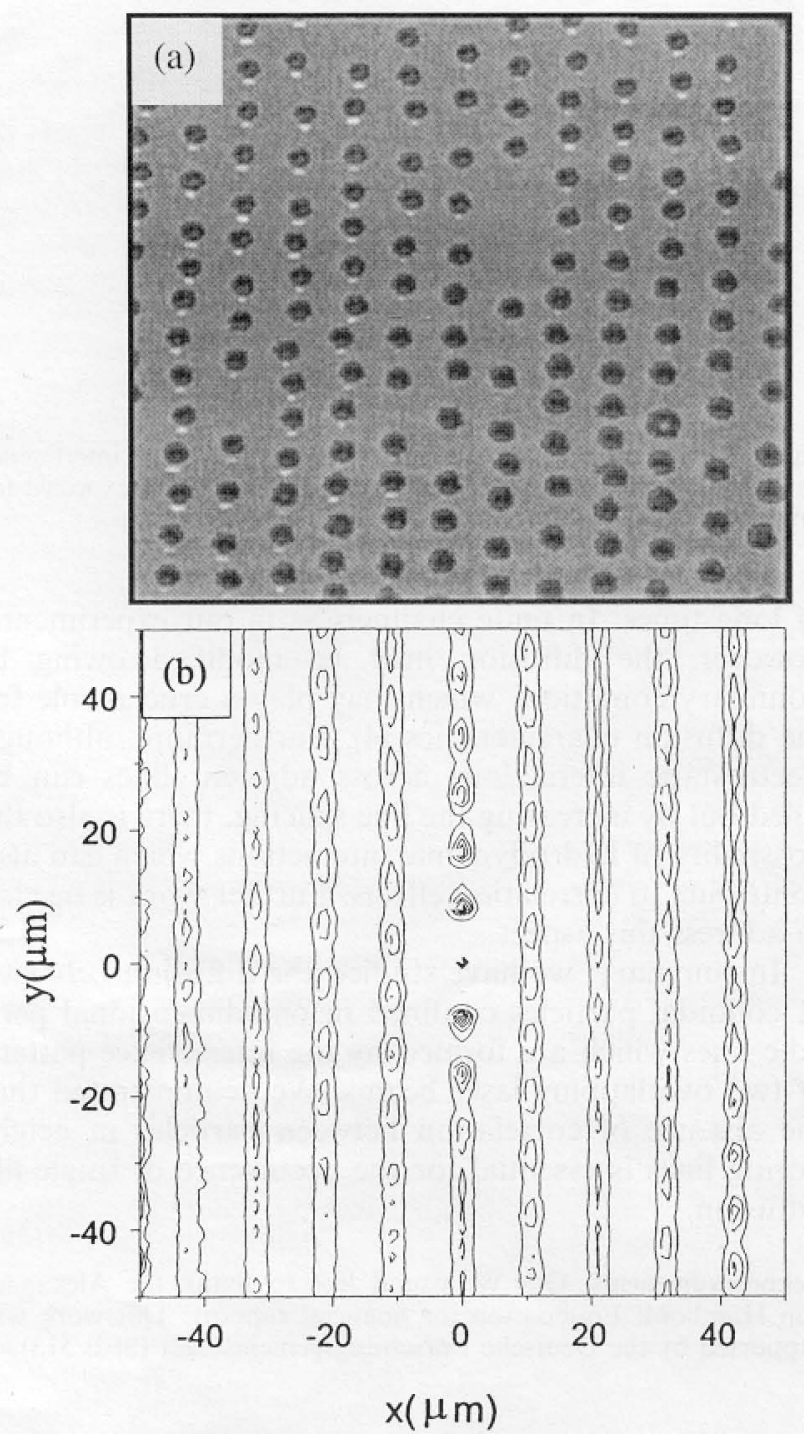

Fig. 1 a Snapshot of the real space particle configuration of a twodimensional colloidal suspension in the presence of a modulated light field, which localizes the particles along vertical lines. $\mathbf{b}$ The particle pair distribution function corresponding to the particle concentration of a. About 200 real space pictures were used to obtain this plot

$\left\langle\delta y^{2}\right\rangle$ increases linearly with time, being typical for diffusion in two and three dimensions. From the slopes we obtained the long-time self-diffusion coefficients $D_{\mathrm{L}}$ to be $0.09 \mu \mathrm{m}^{2} / \mathrm{s}$ and $0.02 \mu \mathrm{m}^{2} / \mathrm{s}$ for low and high particle concentrations, respectively. The fact that $\left\langle\delta y^{2}\right\rangle$ increases linearly with time demonstrates that we were not dealing with single-file diffusion. Obviously, the coupling of particles in adjacent "channels" gives rise to a diffusion behavior which is identical to that in two or three dimensions.

In the following we investigate the diffusion behavior when the coupling between particles in adjacent lines is

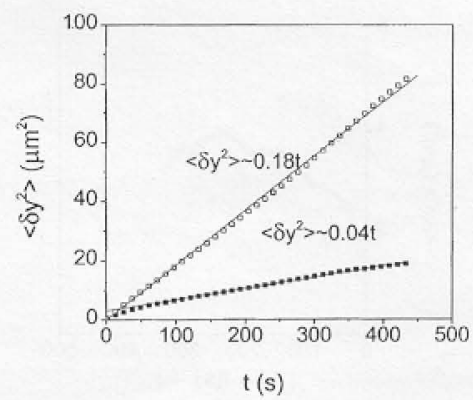

Fig. 2 The particle mean square displacements $\left\langle\delta y^{2}\right\rangle$ along the interference fringes in the case of strongly coupled lines. The open and closed symbols correspond to a system with low and high particle concentrations, respectively

largely reduced. Experimentally this can be achieved by simply increasing the spacing between lines.

Figure 3 a shows a snapshot of a colloidal system where the period of the external potential is increased to about $20 \mu \mathrm{m}$. From the corresponding, averaged twodimensional $g(x, y)$ plot it can be seen that, at such large line spacings, almost no correlation in adjacent lines is visible.

\begin{tabular}{|c|c|c|c|c|c|c|c|}
\hline \multirow[t]{2}{*}{ (a) } & 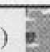 & 8 & $\Rightarrow$ & क & & 9 & \\
\hline & 6 & a & 4 & 9 & 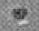 & +8 & $\Rightarrow$ \\
\hline 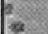 & $=$ & $\theta$ & i) & $\theta$ & $\Leftrightarrow$ & 6 & \\
\hline 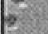 & 8 & a) & 9 & 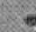 & $\Leftrightarrow$ & it & 8 \\
\hline & 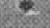 & $\%$ & 9 & 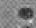 & 6 & $\Leftrightarrow$ & $*$ \\
\hline & e. & 2 & 8 & 6 & 8 & 8 & 4 \\
\hline F & 8 & 9 & 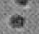 & 8 & 8 & 8 & $\approx$ \\
\hline 2 & $=$ & 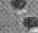 & $\approx$ & 8 & 2 & $\frac{4}{20}$ & 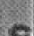 \\
\hline & 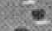 & 8 & 6 & $\infty$ & * & $\theta$ & 6 \\
\hline 3 & 6 & 2 & 0 & $\theta$ & $=$ & 2 & का \\
\hline 45 & 3 & $\omega$ & 42 & 8 & 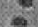 & 4 & 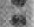 \\
\hline & e. & e. & 2 & $\approx$ & $p$ & 6 & a \\
\hline & & 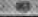 & & & & & \\
\hline
\end{tabular}

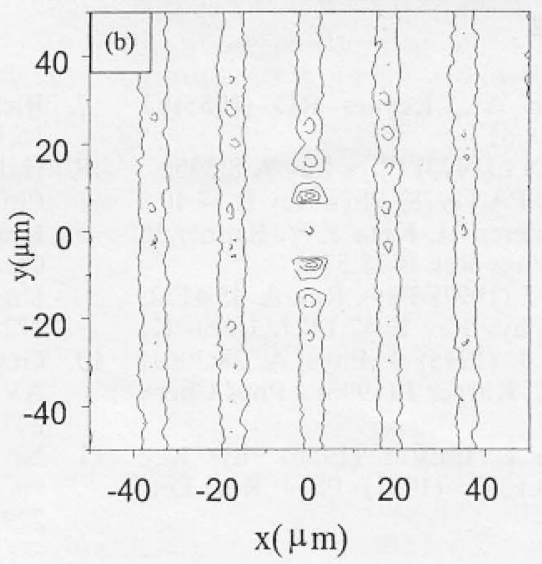

Fig. 3 a Snapshot of the real space particle configuration of a twodimensional colloidal suspension in the presence of a modulated light field. The line spacing is about $20 \mu \mathrm{m}$ and largely reduces the interactions of colloids in adjacent lines. $\mathbf{b}$ The corresponding particle pair distribution function 


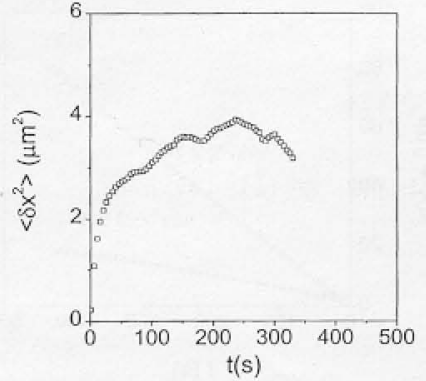

Fig. 4 The particle mean square displacement $\left\langle\delta x^{2}\right\rangle$ perpendicular to the lines demonstrates that the particles are confined in the $x$ direction. The saturation value indicates that no mutual takeover of individual particles within a row is possible

Figure 4 shows the mean square displacement $\left\langle\delta x^{2}\right\rangle$ of the particles perpendicular to the potential lines, which saturates for long times at about $4 \mu \mathrm{m}^{2}$. Consequently, the maximum particle excursion perpendicular to the potential lines is less than the particle diameter and thus, together with the strongly repulsive Coulomb interaction, avoids the mutual takeover of particles within a line. As already mentioned, this is essential for the occurrence of single-file diffusion.

When analyzing the mean square displacement $\left\langle\delta y^{2}\right\rangle$ in this system we find no linear increase but a quite different behavior, as shown in Fig. 5. The solid line corresponds to a fit with a power law $\left\langle\delta y^{2}\right\rangle=b t^{0.5}$, with $b$ being a fit parameter. As can be seen, our data above $t=100 \mathrm{~s}$ can be described by this fit and thus indicates that single-file diffusion can be also observed in colloidal systems under the influence of an external light field.

It should be mentioned that perfect single-file diffusion is only predicted for a very large system in the limit

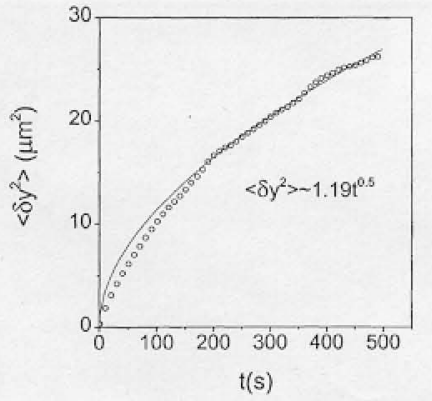

Fig. 5 Mean square displacement $\left\langle\delta y^{2}\right\rangle$ along the interference pattern. The solid line corresponds to a $t^{0.5}$ fit, which is expected for single-file diffusion

of long times. In finite channels as in our experiments, however, the diffusion may be modified owing to boundary conditions which may play a crucial role for the diffusion characteristics [4]. Furthermore, although electrostatic interactions across adjacent lines can be ruled out by increasing the line spacing, there is also the possibility of hydrodynamic interactions which can also contribute to correlation effects. Further work is needed to address this aspect.

In summary, we have studied the diffusion behavior of colloidal particles confined in one-dimensional periodic lines which are formed by the interference pattern of two overlapping laser beams. We demonstrated that the absence of correlation between particles in neighboring lines is essential for the occurrence of single-file diffusion.

Acknowledgements Q.H.W. would like to thank the Alexander von Humboldt Foundation for financial support. This work was supported by the Deutsche Forschungsgemeinschaft (SFB 513).

\section{References}

1. Hodgkin AL, Keynes RD (1955) J Phys 128:61

2. Levitt DG (1973) Phys Rev A 8:3050

3. Fedders PA (1978) Phys Rev B 17:40

4. Van Beijeren H, Kehr KW, Kutner R (1983) Phys Rev B 28:5711

5. Kärger J (1992) Phys Rev A 45:4172; (1993) Phys Rev E 47:1427; Hahn K, Kärger J (1995) J Phys A 28:3061; Hahn K, Kärger J (1996) J Phys Chem 100:316

6. Gunther L, Imry Y (1980) Phys Rev Lett 40:1225; (1981) Phys Rev Lett 46:76
7. Richards PM (1977) Phys Rev B 16:1393

8. Hahn K, Kärger J, Kukla V (1996) Phys Rev Lett 76:2762

9. Kukla V, Kornatowski J, Demuth D, Girnus I, Pfeifer H, Rees L, Schunk S, Unger K, Kärger J (1996) Science 272:702

10. Gupta V, Nivarthi SS, McCormick AV, Davis HT (1995) Chem Phys Lett 247:596

11. Nivarthi SS, McCormick AV, Davis HT (1994) Chem Phys Lett $229: 297$
12. Jobic H, Hahn K, Kärger J, Bee M, Tuel A, Noack M, Girnus I, Kearley GJ (1997) J Phys Chem 110:5834

13. Radhakrishnan R, Gubbins KE (1998) Phys Rev Lett 79:2847

14. Löwen H (1994) Phys Rep 237:249

15. Wei Q-H, Bechinger C, Rudhardt D, Leiderer P (1998) Phys Rev Lett 81:2606

16. Loudiyi K, Ackerson BJ (1992) Physica A $184: 26$

17. Ashkin A (1970) Phys Rev Lett 24:156; Ashkin A, Dziedzic JM (1975) Science 187:1073 